\title{
ON SPHERICAL INVERSIONS OF POLYHARMONIC FUNCTIONS*
}

\author{
BY \\ ALLEN T. CHWANG \\ Institute of Hydraulic Research, The University of Iow'a
}

\begin{abstract}
A general spherical inversion theorem for polyharmonic functions of order $m$ has been obtained, and it reduces to the Kelvin transformation for $m=1$. For biharmonic functions $(m=2)$, the present theorem has been applied to generate an explicit solution which satisfies the prescribed homogeneous boundary conditions on the surface of a sphere.
\end{abstract}

1. Introduction. The spherical inversion theorem for harmonic functions, often known as the Kelvin transformation (see Yih [13], Kellogg [7], and Courant and Hilbert [6]), has been used frequently to determine the image system of a given potential distribution in the presence of a sphere. Applying the Kelvin transformation, Weiss [10] established a relationship connecting the velocity potential of the irrotational flow of an incompressible inviscid fluid around a sphere with that of the flow when the sphere is absent. The two-dimensional counterpart of the Weiss sphere theorem was obtained earlier by MilneThomson [9], while Butler [3] solved the axisymmetric potential flow problem around a sphere in terms of the Stokes stream function. The sphere theorems of Weiss and Butler were subsequently generalized and extended by Ludford et al. [8] and Yeh et al. [11] to cover boundary conditions applicable to the fields of hydrodynamics, heat, magnetism, and electrostatics.

In the study of a slow, steady axisymmetric motion of viscous fluid bounded by a sphere, Collins $[4,5]$ has found the spherical image system, in terms of the Stokes stream function, for a given axisymmetric motion in an unbounded fluid whether the motion is irrotational or not. The governing differential equations for the slow, steady motion of a viscous fluid are the Stokes equations, which require the velocity vector $\mathbf{U}(x, y, z)$ to satisfy the biharmonic equation

$$
\Delta^{2} \mathbf{U}(x, y, z)=0
$$

\footnotetext{
${ }^{*}$ Received September 17, 1985.
} 
where $\Delta$ is the Laplacian operator. In a three-dimensional Euclidean space the Laplacian is defined as

$$
\Delta=\frac{\partial^{2}}{\partial x^{2}}+\frac{\partial^{2}}{\partial y^{2}}+\frac{\partial^{2}}{\partial z^{2}} .
$$

Burns [2] extended Milne-Thomson's [9] circle theorem to the case where a polyharmonic function is constructed which satisfies the homogeneous boundary conditions on the circle and has the same singularities outside the circle as the given harmonic function which satisfies the Laplace equation (1). A function $U(x, y, z)$ is said to be a polyharmonic function of order $m$ in a domain $D$ of a three-dimensional Euclidean space if it satisfies an elliptic differential equation of the form

$$
\Delta^{m} U=0 \quad(m=1,2,3, \ldots)
$$

throughout the domain $D$, where $\Delta$ is the Laplacian operator given by Eq. (2). For $m=1$, the differential equation (3) reduces to the Laplace equation, and its solutions are simply called harmonic functions, or harmonics. For $m=2$, Eq. (3) is often called the biharmonic equation, and its solutions biharmonics. It is obvious that a polyharmonic function of order $m$ satisfies a set of differential equations $\Delta^{k} U=0$, with $k \geqslant m, k$ being a positive integer.

The objective of the present paper is to provide a general inversion transformation with respect to a sphere for a given polyharmonic function of order $m$. The resulting function and the original function satisfy the same differential equation of the type given by Eq. (3).

2. Inversion theorem for polyharmonic functions. Before we introduce the general inversion theorem for polyharmonic functions with respect to a sphere, we first establish some relationships between polyharmonic functions of order $m$ and harmonics $(m=1)$.

Lemma 1. A polyharmonic function of order $m, U(x, y, z)$, can be represented by a sum of products of harmonic functions and powers of $r^{2}\left(r^{2}=x^{2}+y^{2}+z^{2}\right)$, that is

$$
U(x, y, z)=\sum_{i=1}^{m} r^{2(i-1)} \phi_{i}(x, y, z),
$$

where the $\phi_{i}$ 's satisfy the Laplace equations

$$
\Delta \phi_{i}(x, y, z)=0 \quad(i=1,2, \ldots, m) .
$$

Proof. Since $U(x, y, z)$ is a polyharmonic function of order $m$, it satisfies, by definition, the elliptic differential equation $\Delta^{m} U=0$. Let $V(x, y, z)$ denote $\Delta^{m-1} U(x, y, z)$. Then $V$ is a harmonic function since $\Delta V=\Delta\left(\Delta^{m-1} U\right)=\Delta^{m} U=0$. Any harmonic function may be expanded in series of spherical harmonics (Kellogg [7]). Hence we have

$$
\Delta^{m-1} U=V=\sum_{n=-\infty}^{\infty} a_{n} H_{n}(x, y, z)
$$

where the $a_{n}$ 's are constants, $H_{n}(x, y, z)$ is a spherical harmonic of degree $n$, and

$$
H_{-(n+1)}(x, y, z)=r^{-(2 n+1)} H_{n}(x, y, z) \quad(n=0,1,2, \ldots) .
$$


The solution of Eq. (6) consists of two parts: a complementary solution $U_{c}$ which satisfies the homogeneous equation $\Delta^{m-1} U_{\mathrm{c}}=0$, and a particular integral $U_{\mathrm{p}}$ which satisfies Eq. (6) itself. The particular integral can be found by inspection to be

$$
U_{\mathrm{p}}=r^{2(m-1)} \phi_{m},
$$

where

$$
\phi_{m}(x, y, z)=\sum_{n=-\infty}^{\infty} \frac{a_{n} H_{n}(x, y, z)}{2^{m-1}(m-1) !(2 n+2 m-1)(2 n+2 m-3) \cdots(2 n+3)}
$$

and $\Delta \phi_{m}=0$. In obtaining Eqs. (7a) and (7b), we have applied Euler's relation for a homogeneous function of degree $n$ such that

$$
x \frac{\partial H_{n}}{\partial x}+y \frac{\partial H_{n}}{\partial y}+z \frac{\partial H_{n}}{\partial z}=n H_{n} .
$$

Hence,

$$
U(x, y, z)=U_{\mathrm{c}}(x, y, z)+r^{2(m-1)} \phi_{m}(x, y, z) .
$$

As $U_{\mathrm{c}}$ satisfies the equation $\Delta^{m-1} U_{\mathrm{c}}=0$, it is a polyharmonic function of order $(m-1)$.

We can repeat the above process $(m-2)$ times and obtain the expansion for $U(x, y, z)$ as

$$
U(x, y, z)=\sum_{i=1}^{m} r^{2(i-1)} \phi_{i}(x, y, z)
$$

with

$$
\Delta \phi_{i}(x, y, z)=0 \quad(i=1,2, \ldots, m) .
$$

We should note that Lemma 1 can also be proved by successively applying the Almansi theorem [1]. In the present notation, the Almansi theorem states that if $\phi_{m-1}(x, y, z)$ is a polyharmonic function of order $m-1$, then $r^{2} \phi_{m-1}$ is a polyharmonic function of order $m$. It is of interest to also note that Lemma 1 is similar to, but not the same as, Yih's theorem [12] for the hyper-Bessel equation $L_{p}^{m} f=0$, where $L_{p} \equiv D^{2}+r^{-1} D-p^{2} r^{-2}+k^{2}$ with $D \equiv d / d r$. Yih's theorem states that the solutions of the hyper-Bessel equation are $r^{n} Z_{p+n}(k r)$ in which $n=0,1,2, \ldots, m-1$, and $Z_{p}(k r)$ stands for the Bessel function of the first kind or the second kind, $L_{p} Z_{p}(k r)=0$.

By direct calculation and application of Lemma 1, we can easily establish the following results regarding a polyharmonic function of order $m$.

Lemma 2. If $\phi(x, y, z)$ is a harmonic function, then the function $r^{2(m-1)} \phi(x, y, z), m$ being a positive integer, is a polyharmonic function of order $m$.

LEMMA 3. If $U$ is a polyharmonic function of order $m, \Delta^{m} U=0$, then the function $V=r \partial U / \partial r$ is also a polyharmonic function of the same order $m$, that is $\Delta^{m}(r \partial U / \partial r)=0$.

LEMma 4. If $U$ is a polyharmonic function of order $m, \Delta^{m} U=0$, then $r^{2 h} U$ is a polyharmonic function of order $(k+m)$, namely $\Delta^{k+m}\left(r^{2 k} U\right)=0$, both $m$ and $k$ being positive integers. 
With the above lemmas established, we may now introduce the general inversion theorem for polyharmonic functions of order $m$.

THEOREM. If $U(x, y, z)$ is a polyharmonic function of order $m$ ( $m$ being a positive integer) in a domain $D$ of the three-dimensional Euclidean space, then the function

$$
V(x, y, z)=r^{2 m-3} U\left(\frac{x}{r^{2}}, \frac{y}{r^{2}}, \frac{z}{r^{2}}\right) \quad\left(r^{2}=x^{2}+y^{2}+z^{2}\right)
$$

is also a polyharmonic function of the same order $m$ in the domain $D^{\prime}$ obtained from $D$ by inversion with respect to the unit sphere.

Proof. We first note that if $U(x, y, z)$ is a regular harmonic function $(m=1)$, this transformation reduces to the well-known Kelvin transformation. The proof of the Kelvin transformation can be found, for example, in Kellogg's [7] or Courant and Hilbert's [6] book.

For arbitrary values of $m$, we have from Lemma 1 that

$$
U(x, y, z)=\sum_{i=1}^{m} r^{2(i-1)} \phi_{i}(x, y, z),
$$

where $\phi_{i}$ is a harmonic function. After the inversion, it follows from Eqs. (9) and (10) that

$$
\begin{aligned}
V(x, y, z) & =r^{2 m-3} \sum_{i=1}^{m} r^{-2(i-1)} \phi_{i}\left(\frac{x}{r^{2}}, \frac{y}{r^{2}}, \frac{z}{r^{2}}\right) \\
& =\sum_{i=1}^{m} r^{2(m-i)}\left[\frac{1}{r} \phi_{i}\left(\frac{x}{r^{2}}, \frac{y}{r^{2}}, \frac{z}{r^{2}}\right)\right] .
\end{aligned}
$$

Let

$$
\psi_{i}(x, y, z)=\frac{1}{r} \phi_{i}\left(\frac{x}{r^{2}}, \frac{y}{r^{2}}, \frac{z}{r^{2}}\right) \quad(i=1,2, \ldots, m) .
$$

By the Kelvin transformation for harmonic functions, we know that the functions $\psi_{i}(x, y, z)(i=1,2, \ldots, m)$ are also harmonics.

Applying Lemma 2 and noting that a polyharmonic function of order $n$ satisfies a set of differential equations $\Delta^{k} U=0(k \geqslant n)$, we obtain

$$
\Delta^{m} V(x, y, z)=\Delta^{m}\left[\sum_{i=1}^{m} r^{2(m-i)} \psi_{i}(x, y, z)\right]=0 .
$$

In other words, $V(x, y, z)$ given by Eq. (9) is a polyharmonic function of order $m$.

3. Extension of the inversion theorem. The inversion theorem given in the previous section can be extended to $n$ dimensions. Thus, if $U\left(x_{1}, x_{2}, \ldots, x_{n}\right)$ is a polyharmonic function of order $m$ in a domain $D$ of an $n$-dimensional $x$-space, then the function

$$
V=r^{2 m-n} U\left(\frac{x_{1}}{r^{2}}, \frac{x_{2}}{r^{2}}, \ldots, \frac{x_{n}}{r^{2}}\right) \quad\left(r^{2}=\sum_{i=1}^{n} x_{i}^{2}\right)
$$

is also a polyharmonic function of the same order $m$ in the domain $D^{\prime}$ obtained from $D$ by inversion with respect to the unit sphere. The proof of this extended theorem is similar 
to that of the original theorem with the $\psi_{i}$ 's in Eq. (12) replaced by

$$
\psi_{i}\left(x_{1}, x_{2}, \ldots, x_{n}\right)=r^{2-n} \phi_{i}\left(\frac{x_{1}}{r^{2}}, \frac{x_{2}}{r^{2}}, \ldots, \frac{x_{n}}{r^{2}}\right) \quad(i=1,2, \ldots, m) .
$$

We note that Lemmas 1-4 remain the same in $n$-dimensional space except that the Laplacian operator becomes

$$
\Delta=\sum_{i=1}^{n} \frac{\partial^{2}}{\partial x_{i}^{2}}
$$

Finally, we remark that if the inversion is made with respect to a sphere of radius $a$, the dimensional form of Eq. (13) is

$$
V=\left(\frac{r}{a}\right)^{2 m-n} U\left(\frac{a^{2} x_{1}}{r^{2}}, \frac{a^{2} x_{2}}{r^{2}}, \ldots, \frac{a^{2} x_{n}}{r^{2}}\right), \quad\left(r^{2}=\sum_{i=1}^{n} x_{i}^{2}\right) .
$$

4. Examples. Suppose that a biharmonic function $f(r, \theta, \phi)$ has all its singularities outside a sphere $r=a,(r, \theta, \phi)$ being the spherical polar coordinates. We seek, in the presence of a sphere $r=a$, a biharmonic function $g(r, \theta, \phi)$ which has the same singularities outside the sphere as $f(r, \theta, \phi)$ and which satisfies the homogeneous boundary conditions

$$
g(a, \theta, \phi)=\frac{\partial}{\partial r} g(a, \theta, \phi)=0 .
$$

By Lemma 1 and Eqs. (16) (with $m=2, n=3$ ), we can write the required biharmonic function $g(r, \theta, \phi)$ in the form

$$
g(r, \theta, \phi)=f(r, \theta, \phi)-\frac{r}{a} f\left(\frac{a^{2}}{r}, \theta, \phi\right)+\left(r^{2}-a^{2}\right) h(r, \theta, \phi),
$$

where $h(r, \theta, \phi)$ is a harmonic function,

$$
\Delta h=\frac{1}{r^{2}} \frac{\partial}{\partial r}\left(r^{2} \frac{\partial h}{\partial r}\right)+\frac{1}{r^{2} \sin \theta} \frac{\partial}{\partial \theta}\left(\sin \theta \frac{\partial h}{\partial \theta}\right)+\frac{1}{r^{2} \sin ^{2} \theta} \frac{\partial^{2} h}{\partial \phi^{2}}=0 .
$$

Obviously $g(r, \theta, \phi)$ given by Eq. (18) satisfies the boundary condition that $g(a, \theta, \phi)=0$. Applying the second boundary condition of Eq. (17) to Eq. (18), we can determine the harmonic function $h(r, \theta, \phi)$ as

$$
a h(r, \theta, \phi)=\frac{1}{2 r} f\left(\frac{a^{2}}{r}, \theta, \phi\right)+\frac{\partial}{\partial r} f\left(\frac{a^{2}}{r}, \theta, \phi\right)-\frac{r^{2}\left(r^{2}-a^{2}\right)}{4 a^{2}} \Delta\left[\frac{1}{r} f\left(\frac{a^{2}}{r}, \theta, \phi\right)\right] .
$$

To show that $h(r, \theta, \phi)$ given by Eq. (19) is indeed a harmonic function, we first express the biharmonic function $f(r, \theta, \phi)$ by Lemma 1 as

$$
f(r, \theta, \phi)=f_{1}(r, \theta, \phi)+\left(r^{2} / a^{2}\right) f_{2}(r, \theta, \phi),
$$

where both $f_{1}$ and $f_{2}$ are harmonic functions. Substituting Eq. (20) into Eq. (19), we have

$$
a h(r, \theta, \phi)=\frac{\partial}{\partial r} f_{1}\left(\frac{a^{2}}{r}, \theta, \phi\right)+\frac{\partial}{\partial r} f_{2}\left(\frac{a^{2}}{r}, \theta, \phi\right)+\frac{1}{2 r} f_{1}\left(\frac{a^{2}}{r}, \theta, \phi\right)-\frac{3}{2 r} f_{2}\left(\frac{a^{2}}{r}, \theta, \phi\right) \text {. }
$$


Since $f_{1}(r, \theta, \phi)$ and $f_{2}(r, \theta, \phi)$ are harmonic functions, the last two terms in Eq. (21) are also harmonic functions by Eq. (16) with $m=1, n=3$. By Lemma 3, we know that

$$
r \frac{\partial}{\partial r}\left[\frac{1}{r} f_{1}\left(\frac{a^{2}}{r}, \theta, \phi\right)\right]
$$

is a harmonic function,

$$
0=\Delta\left\{r \frac{\partial}{\partial r}\left[\frac{1}{r} f_{1}\left(\frac{a^{2}}{r}, \theta, \phi\right)\right]\right\}=\Delta\left[\frac{\partial}{\partial r} f_{1}\left(\frac{a^{2}}{r}, \theta, \phi\right)\right] .
$$

Therefore the first term on the right-hand side of Eq. (21) is a harmonic function. Similarly the second term on the right-hand side of Eq. (21) is a harmonic function. Hence the function $h(r, \theta, \phi)$ given by Eq. (19) is indeed a harmonic function.

Finally, substituting Eq. (19) into Eq. (18), we obtain that

$$
\begin{aligned}
g(r, \theta, \phi)=f( & (r, \theta, \phi)-\frac{\left(r^{2}+a^{2}\right)}{2 a r} f\left(\frac{a^{2}}{r}, \theta, \phi\right)+\frac{\left(r^{2}-a^{2}\right)}{a} \frac{\partial}{\partial r} f\left(\frac{a^{2}}{r}, \theta, \phi\right) \\
& -\frac{r^{2}\left(r^{2}-a^{2}\right)^{2}}{4 a^{3}} \Delta\left[\frac{1}{r} f\left(\frac{a^{2}}{r}, \theta, \phi\right)\right] .
\end{aligned}
$$

By direct calculation we can verify that the biharmonic function $g(r, \theta, \phi)$ given by Eq. (22) also satisfies the boundary condition that $(\partial / \partial r) g(a, \theta, \phi)=0$. As $f(r, \theta, \phi)$ has all its singularities outside the sphere $r=a, f\left(a^{2} / r, \theta, \phi\right)$ must have all its singularities inside the sphere $r=a$. Hence the function $g(r, \theta, \phi)$ given by Eq. (22) is the required biharmonic function which has the same singularities outside the sphere $r=a$ as $f(r, \theta, \phi)$ and which satisfies the homogeneous boundary conditions of Eq. (17).

In general, if $f(r, \theta, \phi)$ is a polyharmonic function of order $m, m$ being a positive integer, and $f(r, \theta, \phi)$ has all its singularities outside a sphere $r=a$, then the function $g(r, \theta, \phi)$, which is also a polyharmonic function of the same order $m$ as $f(r, \theta, \phi)$, has the same singularities outside the sphere as $f(r, \theta, \phi)$ and satisfies the homogeneous boundary conditions

$$
g(a, \theta, \phi)=\frac{\partial}{\partial r} g(a, \theta, \phi)=\cdots=\frac{\partial^{m-1}}{\partial r^{m-1}} g(a, \theta, \phi)=0,
$$

which can be expressed as

$$
g(r, \theta, \phi)=f(r, \theta, \phi)-\left(\frac{r}{a}\right)^{2 m-3} f\left(\frac{a^{2}}{r}, \theta, \phi\right)+\sum_{k=1}^{m-1}\left(r^{2}-a^{2}\right)^{k} h_{k}(r, \theta, \phi),
$$

where the $h_{k}$ 's are harmonic functions. By Lemma 2 and the Inversion Theorem, we know that $g(r, \theta, \phi)$ given by Eq. (23) is a polyharmonic function of order $m$. It is also evident from Eq. (23) that $g(a, \theta, \phi)=0$. Applying the boundary condition $(\partial / \partial r) g(a, \theta, \phi)=0$, we may determine $h_{1}(r, \theta, \phi)$ in terms of $f\left(\frac{a^{2}}{r}, \theta, \phi\right)$ as we did for the biharmonic function case. Then applying the boundary condition $\left(\partial^{2} / \partial r^{2}\right) g(a, \theta, \phi)=0$, we may determine the harmonic function $h_{2}(r, \theta, \phi)$ in terms of $f\left(\frac{a^{2}}{r}, \theta, \phi\right)$ and so forth until we determine all the $h_{k}$ 's $(k=1,2, \ldots, m-1)$. 
For nonhomogeneous boundary conditions such as

$g(a, \theta, \phi)=u_{0}(\theta, \phi), \quad \frac{\partial}{\partial r} g(a, \theta, \phi)=u_{1}(\theta, \phi), \ldots, \frac{\partial^{m-1}}{\partial r^{m-1}} g(a, \theta, \phi)=u_{m-1}(\theta, \phi)$,

we may assume $g(r, \theta, \phi)$ to have the form

$$
g(r, \theta, \phi)=f(r, \theta, \phi)-\left(\frac{r}{a}\right)^{2 m-3} f\left(\frac{a^{2}}{r}, \theta, \phi\right)+\sum_{k=0}^{m-1}\left(r^{2}-a^{2}\right)^{k} h_{k}(r, \theta, \phi) .
$$

Then the boundary condition $g(a, \theta, \phi)=u_{0}(\theta, \phi)$ will lead to the determination of the harmonic function $h_{0}(r, \theta, \phi)$, the boundary condition $(\partial / \partial r) g(a, \theta, \phi)=u_{1}(\theta, \phi)$ will lead to the determination of the harmonic function $h_{1}(r, \theta, \phi)$, and so forth. However, we shall not pursue this general case any further here.

The author is grateful to the referee for his comments on the Almansi theorem and on Yih's theorem.

\section{REFERENCES}

[1] E. Almansi, Sull'integrazione dell' equazione differenziale $\Delta^{2 n}=0$, Ann. Mat. Pura Appl. (3) 2, 1-51 (1899)

[2] J. C. Burns, A generalization of Milne-Thomson's circle theorem, J. Math. Phys. Sci. 7, 373-382 (1973)

[3] S. F. J. Butler, A note on Stokes's stream function for motion with a spherical boundary, Proc. Cambridge Philos. Soc. 49, 169-174 (1953)

[4] W. D. Collins, A note on Stokes's stream function for the slow steady motion of viscous fluid before plane and spherical boundaries, Mathematika 1, 125-130 (1954)

[5] W. D. Collins, Note on a sphere theorem for the axisymmetric Stokes flow of a viscous fluid, Mathematika 5. 118-121 (1958)

[6] R. Courant and D. Hilbert, Methods of mathematical physics, Vol. 2, Interscience, pp. 242-243 and 286-290 (1962)

[7] O. D. Kellogg, Foundations of potential theory, J. Springer, Berlin, pp. 231-233 and 251-253, 1929

[8] G. S. S. Ludford, J. Martinek, and G. C. K. Yeh, The sphere theorem in potential theory, Proc. Cambridge Philos. Soc. 51, 389-393 (1955)

[9] L. M. Milne-Thomson, Hydrodynamical images, Proc. Cambridge Philos. Soc. 36, 246-247 (1940)

[10] P. Weiss, On hydrodynamical images-arbitrary irrotational flow disturbed by a sphere, Proc. Cambridge Philos. Soc. 40, 259-261 (1944)

[11] G. C. K. Yeh, J. Martinek, and G. S. S. Ludford, A general sphere theorem for hydrodynamics, heat, magnetism, and electrostatics, Z. Angew. Math. Mech. 36, 111-116 (1956)

[12] C. S. Yih, Solutions of the hyper-Bessel equation, Quart. Appl. Math. 13, 462-463 (1956)

[13] C. S. Yih, Fluid mechanics, McGraw-Hill, Inc., pp. 96-97 (1969) 